\title{
PARTICULARITIES OF EXPERIMENTAL MODELS USED TO INDUGE GASTRIC ULCER
}

\section{Simona Fulga', Ana-Maria Pelin², Gristina Mihaela Ghiciuc', Elena Gătălina Lupușoru1}

${ }^{1}$ University of Medicine and Pharmacy "Grigore T. Popa" Iasi, Romania;

${ }^{2}$ Faculty of Medicine and Pharmacy "Dunărea de Jos" Galati, Romania.

Cristina Mihaela Ghiciuc

Department of Pharmacology, Faculty of Medicine,

"Grigore T. Popa" University of Medicine and Pharmacy Iasi, Romania email: cristina.ghiciuc@umfiasi.ro

\begin{abstract}
Introduction: Gastric ulcer is one of the most common gastrointestinal diseases, therefore the constant interest for new treatments is due to adverse effects induced by current therapy. The restricted number of in vivo experimental models is a challenge for researchers. Objectives: Identifying the particularities of different types of experimentally induced gastric ulcer in laboratory animals to facilitate their choise for the study of new antiulcer drugs. Material and method: A search in PubMed and Scopus using keywords ("experimentally" AND "gastric ulcer" AND "rats/mice") to include experimental studies with the description of local-induced changes. Review articles and in vitro studies were excluded. Results and discussions: Experimental researches on new drugs for gastric ulcer use chemical or surgical methods to induce gastric lesions in rats. Non-steroidal anti-inflammatory drugs (NSAIDs) and acetic acid models to investigate antisecretory and cytoprotective effects; ethanol models evaluate cytoprotective and/or antioxidant effects; pylorus ligature models to evaluate the effects on the secretion of aggressive gastric factors (hydrochloric acid or pepsin). NSAIDs (indomethacin, acetylsalicylic acid or ibuprofen) inhibit cyclooxygenase activity, resulting from reduced mucus and bicarbonate secretion, decreased mucosal blood flow, alteration of microvascular structures, causing epithelial damage Ethanol enhances the proteolytic and hydrolytic action of hydrochloric acid and pepsin; in addition, stimulates the acid secretion and disruptes vascular endothelium. Pylorus ligature determines the accumulation of gastric acid resulting in gastric ulcers due to the autodigestion of the mucosa. Conclusion: The knowledge of the mechanisms to induce experimental gastric ulcers is essential for choosing the model to evaluate new antiulcer agents.
\end{abstract}

Keywords: gastric ulcer, experimental models, drug-induced ulcers.

\section{Introduction}

Gastric ulcer, one of the most important gastro-intestinal illnesses, affects the population all over the world. Although there are treatments in medical practice, due to the adverse effects that may occur, their use has become quite limited. The increased interest in new anti-ulcerous therapies has made it necessary to use experimental models of ulcers induced in different laboratory animal species.

The gastric ulcer may be induced by various methods (exposure to various stress factors, pharmacological or surgical) on laboratory animals. There are few experimental models of ulcers, but there are limitations for the use of these models to progress the research of wellconducted therapy în this area of diagnostic.

So considering all, it is useful to revise some experimental models from the literature witch are used to screening the agents with anti-ulcerous potential and to explain their biochemical background so it will easy their contribution to the prevention and the cure of the gastric ulcer.

\section{Material si method}

There was a search in database platforms (PubMed, Scopus), using keywords 
„experimentally” AND ,gastric ulcer” AND „laboratory rats/mice”. Experimental studies were included; review articles and in vitro studies were excluded. Types of local changes induced experimentally were noted.

\section{Results}

The majority of experiments for the study of drugs useful for the treatment of gastic ulcer are carried out on rats or on mice. There are different methods to induce experimentally gastric ulcer, such as:

1. Gastric ulcers through Water Immersion Restraint stress (WRS) resemble the gastric mucosa lesions induced by acute invasive factors (trauma or surgery) or by infection (1). This model was wide admitted for the research of stress ulcers $(2,3)$. The pathological basis of stress-induced gastric ulcer is complex and involve multiple factors, such as inflammation and oxidative stress (1). The model is extensively used and is useful for estimating or studying the effects of the new drugs in the healing process of gastric ulcers in laboratory animals. In the study of Guo et al. (2012), male adult Wistar rats were subjected to combined stress factors: free swimming, simple restraint, conscious or under anesthesia WRS, non-water fluid immersion, immersion without water contact, or place in a cage surrounded by sand. Some rats had their eyes occluded to evaluate the sensitivity structures with a role in the stress response beside skin stimuli (4). The gastric lesions, one or multiple, of rats exposed to WRS were time-dependently (measured up to 7 hours), being more severe when the time exposure was longer (5). This experiment suggested that these are patterns of induction for stress ulcer used in the research activity of anti-ulcerous substances.

\section{Chemically-induced Gastric ulcers}

2.1. Gastric ulcers induced by nonsteroidal anti-inflammatory drugs(NSAIDS)

This class of drugs is known to induce ulcer by blocking prostaglandin synthetase in the cyclooxygenase pathway (6) and preventing the synthesis of prostaglandins, prostacyclins, and thromboxane. The pathophysiology of gastric injuries as a result of the action of NSAIDs involved their ability to reduce prostaglandin (PG) production through the inhibition of COX. PGs have an important role in the modulation of the gastric mucosa integrity through the stimulation of the secretion of bicarbonate and mucus, the maintainance of the blood flow of the mucosa, and regulation of the mucosal cell renewal. PGs have an important role also in the functions of the digestive tract. Additionally, tissue oxidation, lipid peroxidation, and apoptosis or programmed cell death are other factors that contribute to the complex pathophysiology of NSAID-induced gastric lesions (7).

In the majority of instances, there are used indomethacin, an indol derivative, and aspirin to cause the membrane injury and subsequently gastric ulcer. These medications are usually administered on a proper path, in a suitable carriage after having fasted the rats for $24-36$ hours. It is used aspirin in oral administration $125-150 \mathrm{mg} / \mathrm{kg}$ body weight in rats and animals are sacrificed after 4 hours (8). When in the experiment it is used indomethacin, $40-100 \mathrm{mg} /$ $\mathrm{kg}$ body weight is administered orally and the ulcers are marked after $4-8$ hours. To establish the dose necessary for the induction of gastric ulcers, a first a pilot evaluation is necessary.

2.2. Gastric ulcers induced by ethanol / hydrochloric acid

Ethanol is considered a risk factor in the pathological modifications from gastric ulcers (9). Ethanol easily passes through the protective gastric mucus due to its solubilizing effect on the mucus, thus favor the exposure of the gastric mucosa to the action of both hydrochloric acid and pepsin (10), which determine the damage of the membrane. In addition, ethanol reduce the blood flow at the level of the vessels from the gastric mucosa, contributing to microvascular lesions. Usually the animals are deprived of food $24 \mathrm{~h}$ before the experiment, but have free access to water up to $4 \mathrm{~h}$ before the experiment. Gastric ulcers are induced with intragastric administration of an ethanol solution of $95-99 \%$ concentration, in a volume of $0.5 \mathrm{~mL} / 100 \mathrm{~g}$ body weight. This experimental model is similar to the acute gastric ulcers developed in humans.

\subsection{Gastric ulcers induced by histamine}

Histamine, a well-known neurotransmitter, is involved in the regulation of stomach acidity, 
influencing the permeability of blood vessels, contraction of muscles and cerebral function. The highest concentration of histamine is found in the skin, lungs, stomach and lower amounts in the brain and heart. There are some factors that mediates gastric ulcer such as the release of histamine, which constitute the basis for the induction of ulcers. In addition, histamine determines reduction in the production of gastric mucus, disturbances of the normal gastric mucosa secretion, of the digestive motility and of the microcirculation. To induce gastric ulcers, histamine phosphate is administered subcutaneously in a dose that vary from $40 \mathrm{mg} /$ $\mathrm{kg}$ body to $100 \mathrm{mg} / \mathrm{kg}$ body, in animals deprived from food for $18 \mathrm{~h}$ before the start of the experiment and the animals are sacrificed after $2 \mathrm{~h}(11)$.

\subsection{Gastric ulcers induced by reserpine}

Reserpine, another indole alkaloid usually used for the treatment of hypertension, has a peripheral action represented by effects on the cholinergic vegetative nervous system that controls the gastrointestinal tract and smooth muscles (12). Reserpine can cause lesions of the gastric mucosa (such as ulcer) due to the increase of gastric secretions, and increase digestive motility (gastric cramps, diarrhea) due to cholinergic effects (13). In the study of Cantarella et al., the ulcer lesions were induced using Reserpine intraperitoneal in a dose of 25 $\mathrm{mg} / \mathrm{kg}$ /day with $0.5 \%$ solution of acetic acid; rats were sacrificed $24 \mathrm{~h}$ later. Reserpine-induced gastric ulcer are considered a result of the release of histamine from the gastric mastocytes, possibly mediated by the cholinergic system.

\subsection{Gastric ulcers induced by serotonin}

Serotonin is a monoamine (5-hydroxytriptamine, 5-HT) derived from tryptophan (an essential amino acid), and synthesized both in serotonergic neurons of the central nervous system where it has role of neurotransmitter, and in the chromaffin cells of the digestive tract. Serotonin effect on the vessels it is to cause vasoconstriction, that reduces blood flow at the level of gastric mucosa, leading to lesions of the mucosa. Serotonin is administered by gastric intubation after food deprivation for $36 \mathrm{~h}$ (14).

2.6. Gastric ulcers induced by dietylditiocarbamate
The Diethyditiocarbamate model is used to study the cytoprotective drugs that might inhibit the production of superoxide and hydroxyl radicals in the gastric mucosa (15). The lesions are induced at the level of the anthral region of the stomach by mobilizing free radicals inducing ulcer lesions (15). The particularity of the model is the subcutaneously administration of dietyltihidocarbamate, in a volume of $1 \mathrm{ml}$ saline solution, in a dose of $800 \mathrm{mg} / \mathrm{kg}$ body, followed by the oral administration of a dose of $1 \mathrm{ml}$ hydrochloric acid.

\subsection{Ulcers induced by Methylene blue}

Methylene blue (administered orally, in a dose of $100 \mathrm{mg} / \mathrm{kg}$ ) produced the intense lesions of the gastric mucosa due to induction of high levels of reactive substances such as thiobarbituric acid, and of $\mathrm{H}+/ \mathrm{K}+$-ATP-ase and superoxide dismutase activities. Moreover, reduction of blood flow to the gastric mucosa, combined with catalase activity, reduce levels of glutathione. Methylene blue activates $\mathrm{H}+/ \mathrm{K}+$ ATP-ase to increase hydrochloric acid secretion and reduces blood flow to the gastric mucosa and produces local oxidative stress radicals involved in the induction of ulcer of the mucosa. Methylene blue induced ulcer is used for the researches on the mechanisms of anti-ulcerous agents (16).

2.8. Gastric ulcers induced by ironascorbic acid

The particularity of this ulcer is the local injection of a mixture of ferrous iron and ascorbic acid in the gastric wall (17). This association induce penetrating ulcer in a dose-dependent manner being mediated by free oxygen radicals. With either ferrous iron or ascorbic acid alone, gastric ulcers did not form.

\section{Gastric ulcers induced by surgical} methods

\subsection{Gastric ulcers induced by ligature of} the Pylorus

The surgically model, pylorus-ligation induces ulcer, is useful as a model for the evaluation of the pharmacological modalities to influence the gastric secretions. The ligature at the level of the pylor increases the volume of the gastric fluid. The resulting injuries result from self-digestion of the mucosa, which leads to the interruption of this physiologic barrier. In summary, an increase in the accumulation of 
hydrochloric acid and of pepsin, after the ligature of the pylorus can cause further digestion of the mucosa. In this experimental model, the pylorus is bound by "Shay" technique under etheric anesthesia according to Shay et al. (1945), in laboratory animals deprived from food for $36 \mathrm{~h}$, but with free access to water except for the last hour before the pylorus ligature. Under anesthesia, a small incision of the abdomen on the middle line under the xiphoid process is realized and the pylorus ligature is further carefully realized to avoid any traction of the pylorus (18).

3.2. Gastric ulcers induced by ischemiareperfusion (IR)

Gastric ulcer induced by ischemiareperfusion (IR) is another model of surgicalinduced gastric ulcer in rat that involves lesions of the muscle mucosa achieved by clamping the celiac artery. Although the erosions of the gastric mucosa occur at $24 \mathrm{~h}$ and $36 \mathrm{~h}$ after ischemiareperfusion, gastric ulcers involving the lesions of the mucous muscles occur in the area of the gastric glands at $48 \mathrm{~h}$ and $72 \mathrm{~h}$ after the initiation of the lesion. This experimental model can be used for the researches on the pathophysiological mechanisms in the development of gastric ulcer and to evaluate new substances for the treatment (19).

\section{Discussions}

In this paper there were evaluated several experimental models of ulcers, which can be used to test potential antiulcer agents. The knowledge of the pathophysiological modifications of every model are important in the selection of the model for researches on the therapeutic potential of new substances for the healing or prevention of gastric ulcer.

There are many modalities (exposure to environmental stress factors, to chemicals or by surgical methods) to induce gastric ulcers in laboratory animals, but for the chemicallyinduced ulcers there are several options (Figure $1)$.

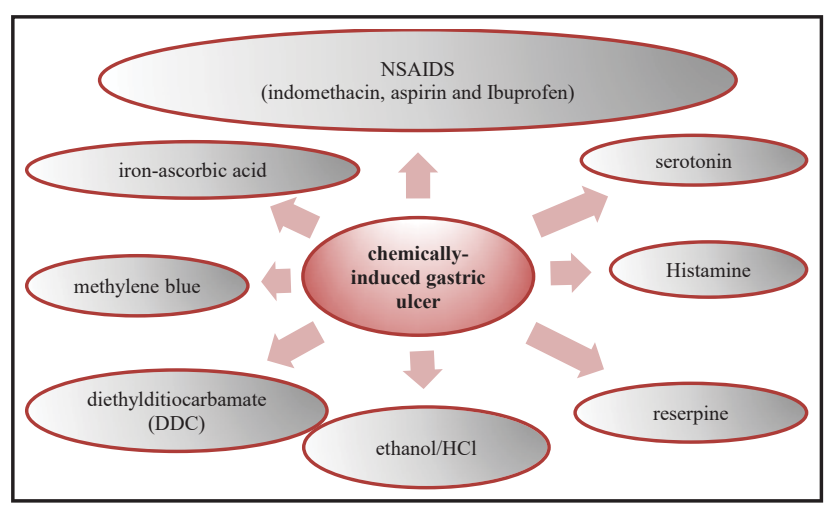

Figure 1. Chemically-induced experimental gastric ulcer.

In our review the water-immersion-andrestraint-stress in rats has been presented as a laboratory model of stress-induced gastric mucosal lesions. It is well known that immersion and / or restraint induce injuries on gastric mucosa, resulting in gastric mucosa erosion, increase of local oxidative stress and the release of high levels of inflammatory factors (20).

The surgical method, pylorus-ligation, is a hypersecretion-induced gastric ulcer model due to pyloric obstruction, followed by subsequent increase in hydrochloric acid and pepsin accumulation, and subsequent mucosal digestion. The pressure receptors in the antral gastric mucosa are stimulated by the high volume of gastric acid secretion, activating the vagus-vagal reflux (21). A high amount of mucus is produced as a result of superficial damage of the mucosa, to favor the repair. This model is used to estimate the cytoprotective effects of new substances supposed to modulate the secretion of gastric mucus. Exposure of gastric mucosa to chemically agents reduces these mucus and bicarbonate secretions, making gastric mucosa more susceptible to destruction action of gastric acid and pepsin.

\section{Conclusions}

The difficulty of the choice of an appropriate experimental model is due to the complexity of disease and to the diversity of the models, each model having strong points and also weak points. The financial resources, the end-point of the study, the hypothesis or the research question that has to be tested influence the final choice of an experimental model. 
The preferred animal models used for researches on new agents for the treatment of gastric ulcer are experimental models based on factors influencing the humans' way of life: ulcer induced by ethanol (22) or NSAIDs (23).

\section{References:}

1. Rau W, Hohaus C, Jessen E. A Differential Approach to Form and Site of Peptic Ulcer. Sci Rep. 2019 Jun 18;9(1):8683.

2. Ernst H, Konturek PC, Brzozowski T, Lochs H, Hahn EG, Konturek SJ. Adaptation of gastric mucosa to stress. Effect of ranitidine. J Physiol Pharmacol. 1998 Sep;49(3):40519.

3. Guo S, Gao Q, Jiao Q, Hao W, Gao X, Cao JM. Gastric mucosal damage in water immersion stress: mechanism and prevention with GHRP-6. World J Gastroenterol. 2012 Jun 28;18(24):3145-55.

4. Rainsford KD. The effects of 5-lipoxygenase inhibitors and leukotriene antagonists on the development of gastric lesions induced by nonsteroidal antiinflammatory drugs in mice. Agents Actions. 1987 Aug;21(3-4):316-9.

5. Nur Azlina M.F., Qodriyah H., Chua K.H., \& Kamisah Y. (2017). Comparison between tocotrienol and omeprazole on gastric growth factors in stress-exposed rats. World J Gastroenterol. 23(32):5887-5894.

6. Somasundaram S, Rafi S, Hayllar J, Sigthorsson G, Jacob M, Price AB, et al. Mitochondrial damage: a possible mechanism of the "topical" phase of NSAID induced injury to the rat intestine. Gut. 1997 Sep;41(3):344-53.

7. Chen XY, Chen HM, Liu YH, Zhang ZB, Zheng YF, Su ZQ, et al. The gastroprotective effect of pogostone from Pogostemonis Herba against indomethacin-induced gastric ulcer in rats. Exp Biol Med (Maywood). 2016 Jan;241(2):193-204.

8. Urushidani T, Kasuya Y, Okabe S. The mechanism of aggravation of indomethacininduced gastric ulcers by adrenalectomy in the rat. Jpn J Pharmacol. 1979 Oct;29(5):77580 .

9. Nazarbahjat N, Kadir FA, Ariffin A, Abdulla MA, Abdullah Z, Yehye WA. Antioxidant
Properties and Gastroprotective Effects of 2-(Ethylthio)Benzohydrazones on EthanolInduced Acute Gastric Mucosal Lesions in Rats. PLoS One. 2016;11(6):e0156022.

10. Ibrahim MY, Hashim NM, Dhiyaaldeen SM, Al-Obaidi MM, El-Ferjani RM, Adam H, et al. Acute Toxicity and Gastroprotection Studies of a New Schiff Base Derived Manganese (II) Complex against $\mathrm{HCl} /$ Ethanol-Induced Gastric Ulcerations in Rats. Sci Rep. 2016 May 27;6:26819.

11. Warzecha Z, Dembinski A, Brzozowski T, Ceranowicz P, Dembinski M, Stachura J, et al. Histamine in stress ulcer prophylaxis in rats. J Physiol Pharmacol. 2001 Sep;52(3):40721.

12. Baumeister A.A., Hawkins M.F. and Uzelac S.M.( 2003): The myth of reserpineinduced depression: role in the historical development of the monoamine hypothesis. J Hist Neurosci 12: 207220.

13. Cheng YT, Lu CC, Yen GC. Phytochemicals enhance antioxidant enzyme expression to protect against NSAID-induced oxidative damage of the gastrointestinal mucosa. Mol Nutr Food Res. 2017 Jun;61(6).

14. Perez S, Talens-Visconti R, Rius-Perez S, Finamor I, Sastre J. Redox signaling in the gastrointestinal tract. Free Radic Biol Med. 2017 Mar; 104:75-103.

15. Oka S, Ogino K, Hobara T, Yoshimura S, Yanai H, Okazaki Y, et al. Role of active oxygen species in diethyldithiocarbamateinduced gastric ulcer in the rat. Experientia. 1990 Mar 15;46(3):281-3

16. Shah DI, Santani DD, Goswami SS. A novel use of methylene blue as a pharmacological tool. J Pharmacol Toxicol Methods. 2006 Nov-Dec;54(3):273-7.

17. Naito $Y$, Yoshikawa $T$, Yoneta $T$, Yagi N, Matsuyama K, Arai M, et al. A new gastric ulcer model in rats produced by ferrous iron and ascorbic acid injection. Digestion. 1995;56(6):472-8.

18. Shay H, Komarov SA, Fels SS, Meranze D, Gruenstein M, Siplet H. A simple method for the uniform production of gastric ulceration in the rat. Gastroenterology. 1945; 5:43-61.

19. Wada K, Kamisaki Y, Kitano M, Kishimoto Y, Nakamoto K, Itoh T. A new gastric ulcer 
model induced by ischemia-reperfusion in the rat: role of leukocytes on ulceration in rat stomach. Life Sci. 1996;59(19):PL295-301.

20. Fu Y, Wu HQ, Cui HL, Li YY, Li CZ. Gastroprotective and anti-ulcer effects of oxymatrine against several gastric ulcer models in rats: Possible roles of antioxidant, antiinflammatory, and prosurvival mechanisms. Phytother Res. 2018 Oct;32(10):2047-58.

21. Adinortey MB, Ansah C, Galyuon I, Nyarko A. In Vivo Models Used for Evaluation of Potential Antigastroduodenal Ulcer Agents. Ulcers. 2013;2013:12.

22. Aziz RS, Siddiqua A, Shahzad M, Shabbir A, Naseem N. Oxyresveratrol ameliorates ethanol-induced gastric ulcer via downregulation of IL-6, TNF-alpha, NF$\mathrm{kB}$, and COX-2 levels, and upregulation of TFF-2 levels. Biomed Pharmacother. 2019 Feb;110:554-60.

23. Piao X, Li S, Sui X, Guo L, Liu X, Li H, et al. 1-Deoxynojirimycin (DNJ) Ameliorates Indomethacin-Induced Gastric Ulcer in Mice by Affecting NF-kappaB Signaling Pathway. Front Pharmacol. 2018;9:372-9. 